\title{
Goal Driven Optimization of Process Parameters for Maximum Efficiency in Laser Bending of Advanced High Strength Steels
}

\author{
Jonathan Griffiths ${ }^{1, a}$, Mike J W Riley ${ }^{1, b}$, Ghazal Sheikholeslami ${ }^{2, c}$, Stuart P \\ Edwardson ${ }^{2, \mathrm{~d}}$ and Geoff Dearden ${ }^{2, e}$ \\ ${ }^{1}$ Laser and Photonics Engineering Group, School of Engineering, University of Lincoln, Brayford \\ Pool, Lincoln, LN6 7TS, United Kingdom \\ ${ }^{2}$ Laser Group, School of Engineering, University of Liverpool, L69 3GH, UK \\ ajgriffiths@lincoln.ac.uk,mriley@lincoln.ac.uk, ’g.sheikholeslami@liv.ac.uk, \\ ds.p.edwardson@liverpool.ac.uk, e'g.dearden@liv.ac.uk
}

Keywords: Forming, Laser, Optimization.

\begin{abstract}
Laser forming or bending is fast becoming an attractive option for the forming of advanced high strength steels (AHSS), due primarily to the reduced formability of AHSS when compared with conventional steels in traditional contact-based forming processes. An inherently iterative process, laser forming must be optimized for efficiency in order to compete with contact based forming processes; as such, a robust and accurate method of optimal process parameter prediction is required. In this paper, goal driven optimization is conducted, utilizing numerical simulations as the basis for the prediction of optimal process parameters for the laser bending of DP 1000 steel. A key consideration of the optimization process is the requirement for minimal microstructural transformation in automotive grade high strength steels such as DP 1000.
\end{abstract}

\section{Introduction}

Of paramount importance in the laser bending process is the selection of suitable process parameters. Process parameters determine the bending mode (that is, towards or away from the beam or in-plane shrinkage), the magnitude of induced bend angle and, crucially in the case of advanced high strength steels (AHSS), the metallurgical characteristics of the workpiece along the irradiation path [1].

The effect of process parameters on the mode of laser bending is well understood. There are three principal mechanisms of laser bending; the buckling mechanism (BM), the upsetting mechanism (UM) and the temperature gradient mechanism (TGM) [2]. Large beam diameters relative to workpiece thicknesses coupled with low traverse speeds typically result in the elasticplastic buckling mechanism being dominant, which can cause deformation towards or away from the beam. The UM is a mechanism of in-plane shortening in which BM process parameters are applied to a workpiece geometry that restricts out of plane bending. The most commonly applied mechanism of laser bending is the TGM due to the relatively high degree of controllability it offers with respect to the other out of plane mechanism of laser bending, the BM. The TGM is characterized by the workpiece bending out of plane towards the beam. This is achieved by inducing a steep thermal gradient locally along the irradiation path, causing the upper material to plastically deform. Upon cooling, providing the temperature was raised enough to cause sufficient thermal strain, contraction of the previously plastically deformed material occurs on this upper surface, yielding a positive bend angle up to approximately $2^{\circ}$ per pass.

An area of limited understanding in laser bending is the effect of process parameters on the metallurgical characteristics of the workpiece along the irradiation path. Undesirable microstructural transformations may result from a combination of peak temperature, time at peak temperature and subsequent cooling rate. Such microstructural transformation could adversely affect the mechanical properties and therefore the subsequent performance of the component; a 
particularly important consideration when processing metallurgically sensitive materials such as AHSS.

Advanced high strength steels such as dual phase (DP) steels play a key role in automotive industry due to reducing weight and crash risk. A key disadvantage of dual phase (DP) steels (such as DP 1000 which forms the basis of this investigation) is their reduced formability when compared with conventional steels. This reduced formability manifests itself in the form of increased spring back. Neugebauer et al. noted that heating of the DP steel is required in order to achieve formability comparable with that of conventional mild steels [3]. However, if the heat treatment is applied uniformly to the entire sheet, the high strength of the steel is severely compromised or lost altogether. Neugebauer et al. proposed a localized laser heat treatment to improve the formability only in regions where it is required for subsequent, contact based forming. Laser forming has the potential to combine both of the aforementioned processes, overcoming the springback issue whilst minimizing the heat affected zone (HAZ) so as to retain the bulk material properties of the steel. Griffiths et al. demonstrated the potential for metallurgically sensitive materials to be formed by the laser bending process, providing optimization of process parameters is conducted [4]. Griffiths identified two temperature regimes over which the high strength of DP 1000 was particularly susceptible to compromise, as characterized by a reduction in hardness. A slight reduction in hardness was observed in the case of low temperature heating regimes, attributed to loss of tetragonality in the martensitic regions of the steel. A relatively larger reduction in hardness was observed for heating regimes in which the maximum temperature exceeded the upper critical transformation temperature for austenitization in mild steel of equivalent carbon content (>1140 K). As such, this loss of hardness was attributed to austenitization of the steel during laser heating.

As a result of the requirement for laser bending of AHSS to operate within strict metallurgical constraints, development of an intelligent predictive system for the determination of suitable process parameters is essential. Research into intelligent predictive systems has hitherto focused on statistical and neural network techniques which use as their basis limited experimental data or simplified analytical models. Safari et al. studied the effect of process parameters on bend angle for mild steel tailor machined blanks [5]. Regression analysis was conducted using experimental data and used to determine the mathematical relationship between process parameters and variation in bend angle, with irradiation path shown to have the greatest influence on bend angle. Maji et al. utilized response surface methodology for modelling and optimization of pulsed laser bending of AISI 304 stainless steel [6]. Statistical regression based on experimental data was used to predict process parameters required for achieving maximum bending angle. Experimental validation of the optimal process parameter set revealed an error in prediction of 4.32\%. Omidvar et al. investigated the optimization of process parameters for maximum bending angle in the laser bending of AA6061-T6 aluminum [7]. A radial basis function neural network utilizing experimental data was used as the basis for a single objective optimization of process parameters which were subsequently validated though experimentation, revealing an error of $<3 \%$ in prediction. Maji et al. applied response surface methodology and statistical regression analysis to the laser forming of dome shapes, comparing optimal process parameters predicted with those predicted using a neural network approach [8]. Upon experimental validation, the process parameters predicted using the neural network approach were found to be more accurate than those predicted using statistical regression, with prediction errors for maximum dome height of $1.46 \%$ and $6.76 \%$ respectively. In a follow-up publication, Maji et al. investigated the application of neural networks and neuro-fuzzy systems in process parameter prediction for the pulsed laser bending process [9]. Whilst utilizing experimental data, Maji et al. noted the potential for incorporation of numerical models; a concept which is of particular relevance to the current work.

In order to achieve optimal efficiency in laser bending with respect to bending mode and magnitude, whilst minimizing micro-structural transformation in the HAZ, a robust, time-effective and cost-effective technique for prediction of suitable process parameters is required. In the current work, a multi-objective genetic algorithm (MOGA) is applied to the goal driven optimization of process parameters for the laser bending process, utilizing transient thermal numerical simulations 
as its basis. A key consideration of the optimization process is the requirement for minimal microstructural transformation in automotive grade DP 1000 high strength steel. The likelihood of microstructural transformation is inferred form simulated temperature.

\section{Methodology}

A goal driven optimization of the laser bending process was conducted, the aim of which was to predict optimal process parameters for maximum efficiency (that is, maximum bend angle) within metallurgical constraints. The optimization process utilized response surfaces generated from thermal finite element simulations of the laser bending process. The component was an $80 \times 80 \mathrm{x}$ $1.5 \mathrm{~mm}$ automotive grade DP1000 steel sheet. An out-of-plane bend towards the beam was required, with the irradiation path along the center of the steel sheet. The laser source was simulated to represent a $1 \mathrm{~kW} \mathrm{CO} \mathrm{TEM}_{00}$ fast axial flow laser with a 3-axis beam delivery system. A schematic of the laser bending process is shown in Fig. 1.

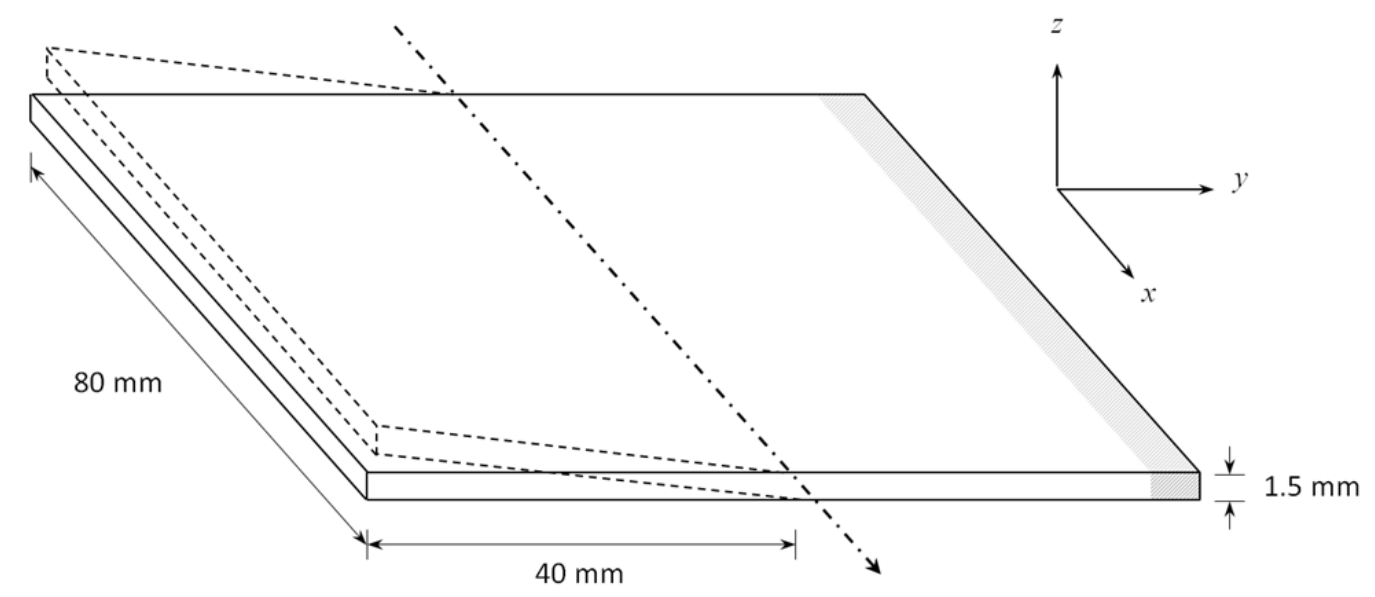

Figure 1: Schematic of the laser bending process where the dashed-dot line represents the irradiation path and direction and the dotted lines represent the laser induced deformation. The shaded area depicts the clamped edge of the component.

Finite element model development. Optimal laser process parameters were predicted for laser bending of $80 \times 80 \times 1.5 \mathrm{~mm}$ DP1000 steel sheet through application of a goal driven optimization approach which used as its basis a numerical model of the laser heating process. A transient-thermal finite element model of the laser bending process was developed in ANSYS Workbench (Version 14). The model incorporated temperature dependent material properties for thermal conductivity, specific heat capacity and density up to $1500 \mathrm{~K}$. The governing equation for conduction in the thermal analysis is:

$$
\rho C_{p} \frac{\delta T}{\delta t}=\nabla \cdot(k \nabla T)
$$

where $\rho$ is the density $\left(\mathrm{kg} / \mathrm{m}^{3}\right), C_{p}$ is the specific heat capacity $(\mathrm{J} / \mathrm{kgK}), T$ is the temperature $(\mathrm{K}), t$ is the time (s) and $k$ is the thermal conductivity $(\mathrm{W} / \mathrm{mK})$. The term $\nabla$ is the differential or gradient operator (sometimes referred to as the Nabla operator) for three dimensional Cartesian co-ordinate systems. All boundaries were subject to convective and radiative heat transfer. Therefore, the heat flux $q$ at a given boundary is given by:

$$
-n \cdot q=-n(-k \nabla T)=q_{0}+h\left(T_{a m b}-T\right)+\varepsilon \sigma\left(T_{a m b}^{4}-T^{4}\right)
$$

where $n$ is the normal vector of the boundary, $h$ is the heat transfer co-efficient $\left(\mathrm{W} / \mathrm{m}^{2} \mathrm{~K}\right), T_{a m b}$ is the ambient temperature (K), $\varepsilon$ is the surface emissivity (1) and $\sigma_{s}$ is the Stefan Boltzmann constant $\left(\mathrm{W} / \mathrm{m}^{2} \mathrm{~K}^{4}\right)$. 
The intensity distribution $I$ of the incident laser beam was approximated by a Gaussian distributed heat source, modelled using ANSYS Parametric Design Language (APDL). The Gaussian heat source is described by Eq. 3:

$$
I=I_{0} e^{-\left(-\frac{2 r^{2}}{\omega_{0}^{2}}\right)}=\frac{2 P}{\pi \omega_{0}^{2}} e^{-\left(-\frac{2 r^{2}}{\omega_{0}^{2}}\right)}
$$

where $I_{0}$ is the peak intensity $\left(\mathrm{W} / \mathrm{m}^{2}\right), P$ is the average laser power $(\mathrm{W}), r$ is radial distance $(\mathrm{m})$ and $\omega_{0}$ is the beam radius $(\mathrm{m})$. An absorption co-efficient of $80 \%$ was specified for all simulations conducted, representative of absorption characteristics of metallic components coated with an absorptive coating such as graphite.

A plane of symmetry was specified along the $z x$ axis in order to reduce the problem size and increase the computational efficiency of the simulation. Globally, the mesh element size was set to fine and determined by automatic meshing in ANSYS. A suitable maximum element size for use along the irradiation path was determined by a convergence study and found to be $0.1 \mathrm{~mm}$. An example output from the model is shown in Fig. 2.

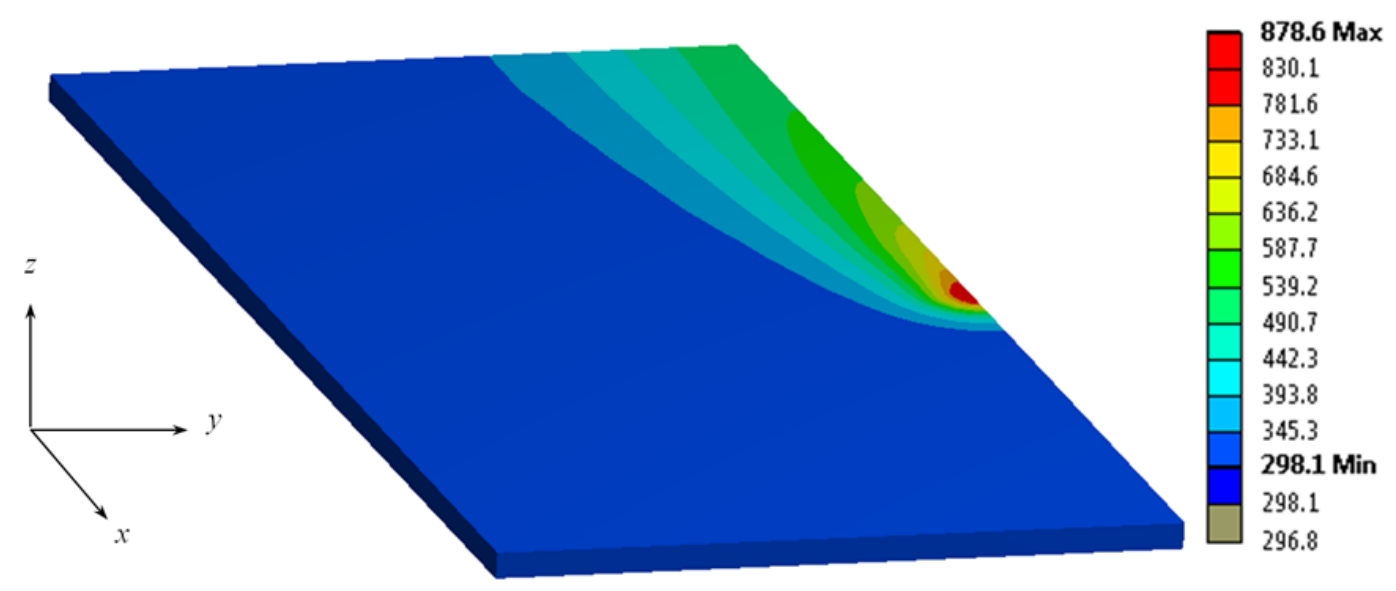

Figure 2: Typical temperature output $(\mathrm{K})$ from the thermal finite element model $(80 \mathrm{x} 80 \mathrm{x} 1.5$ $\mathrm{mm}$ DP 1000, $5.5 \mathrm{~mm}$ spot size, $50 \mathrm{~mm} / \mathrm{s}$ traverse speed, $760 \mathrm{~W}$ laser power, $80 \%$ absorption).

\section{Results and Discussion}

The aim of the numerical work was to determine optimal process parameters for laser bending of a component and focused on (i) determination of the influence of process parameters on maximum simulated temperature and (ii) prediction of optimal process parameters for maximum forming efficiency within metallurgical constraints.

Definition of metallurgical constraints. The characteristic high strength of DP 1000 is particularly susceptible to compromise upon application heat treatment. As such, the laser bending process is required to operate within certain metallurgical constraints in this case. For the purposes of this investigation, the metallurgical constraints were considered the same as those defined by Griffiths et al. [10]. Therefore, the maximum allowable temperature corresponded to the upper critical transformation temperature for austenitization in mild steel of equivalent carbon content (0.15\%wt), in this case $1140 \mathrm{~K}$.

Influence of process parameters. In order to predict optimal process parameters for the laser bending process the complex interrelation of process parameters was characterized through use of finite element simulations. A 31 simulation parametric study was conducted, with design points chosen according to the rotatable central composite design method of design of experiments, the aim of which was to build response surfaces for peak temperature on the top surface of the 
component as a function of process parameters. Three key laser parameters were chosen as input variables for the study; laser power $(P)$, traverse speed $\left(v_{0}\right)$ and spot size $\left(\omega_{0}\right)$. The search domain is given in Table 1, the bounds of which reflect the full range of parameters available given the specification of the laser source intended for use in the subsequent experimental study, as defined in the methodology section.

Table 1: Input process parameters and their ranges for the numerical parametric study.

\begin{tabular}{|lccc} 
Input variable & Initial value & Lower bound & Upper bound \\
$\boldsymbol{P}(\mathbf{W})$ & 550 & 100 & 1000 \\
$\boldsymbol{v}_{\mathbf{0}}(\mathbf{m m} / \mathbf{s})$ & 55 & 10 & 100 \\
$\boldsymbol{\omega}_{\mathbf{0}}(\mathbf{m m})$ & 5.5 & 1 & 10
\end{tabular}
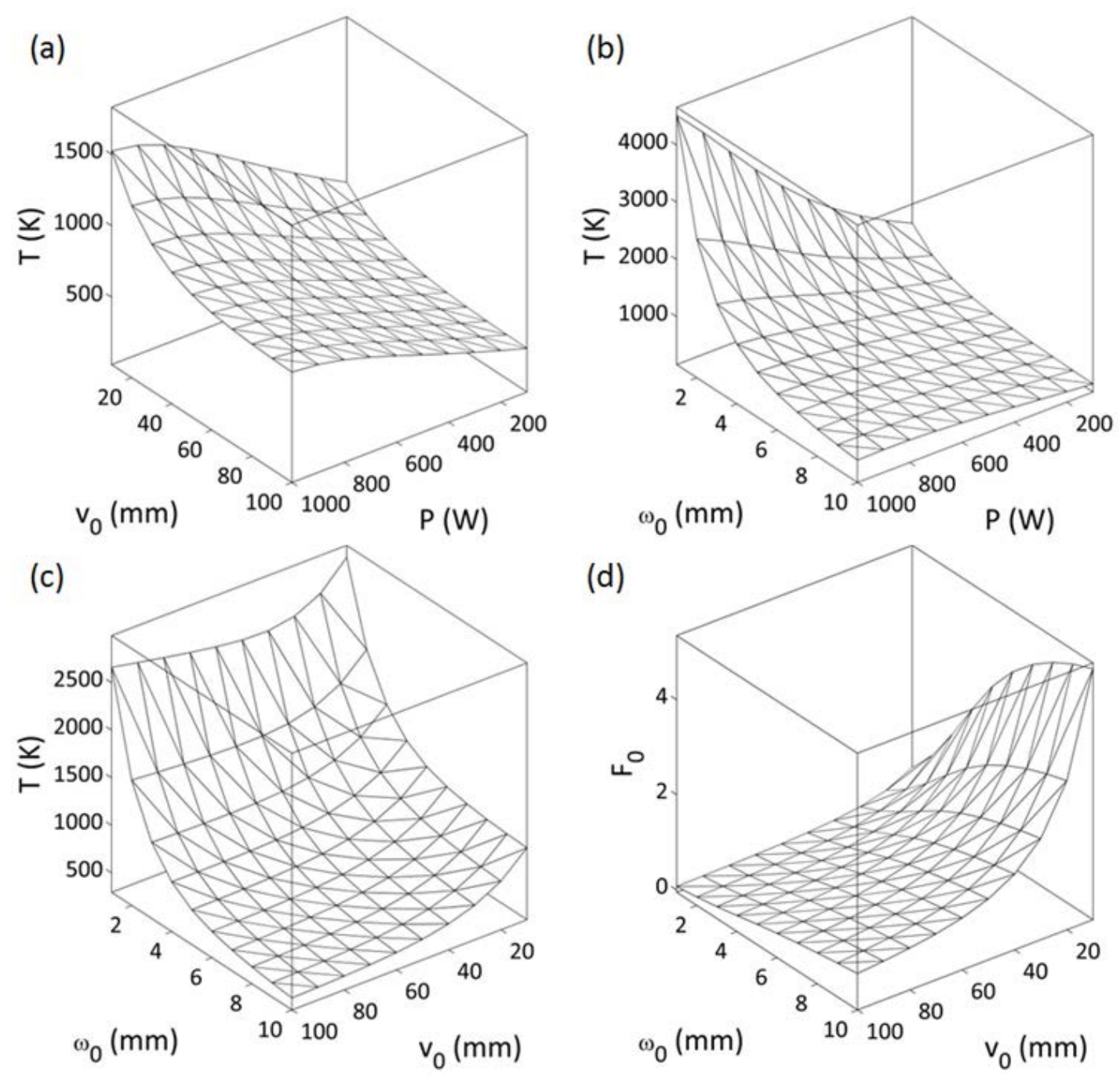

Figure 3: Response surfaces, with (a) maximum temperature as a function of $P$ and $v_{0}\left(\omega_{0}=5.5\right.$ $\mathrm{mm}$ ), (b) maximum temperature as a function of $P$ and $\omega_{0}\left(v_{0}=55 \mathrm{~mm} / \mathrm{s}\right)$, (c) maximum temperature as a function of $v_{0}$ and $\omega_{0}(P=550 \mathrm{~W})$ and $(\mathrm{d})$ Fourier number as a function of $v_{0}$ and $\omega_{0}$.

The output variables for the study were peak transient temperature on the top surface of the component $\left(T_{0}\right)$ and Fourier number $\left(F_{0}\right)$. The latter is a dimensionless number which relates laser traverse speed and spot size to the diffusive thermal properties of the material:

$$
F_{0}=\frac{\alpha_{t h} d_{0}}{v_{0} s_{0}^{2}}
$$


where $\alpha_{t h}$ is the thermal diffusivity ( $\left.\mathrm{m}^{2} / \mathrm{s}\right), d_{0}$ is the beam diameter (m) and $s_{0}$ is the sheet thickness (m) [11]. The Fourier number is a useful quantitative-indication of the likely mechanism of laser bending; that is, TGM, BM or UM. The response surfaces generated by the numerical parametric study are shown in Fig 3.

A useful parameter to consider when analyzing the effect of laser power, traverse speed and spot size on the maximum temperature is the irradiance $\left(I_{r}\right)$, as defined by Eq. 5 , which is dimensionally equivalent to the energy density of the beam.

$$
I_{r}=\frac{P d_{0}}{\pi v_{0} \omega_{0}^{2}}
$$

The values of $I_{r}$ over the range of input variables listed in Table 1 are shown in Fig 4 . In conjunction with Fig. 3 (a-c), Fig. 4 reveals a clear correlation between maximum temperature and laser irradiance. Fig. 4 (a) suggests that parametric combinations which incorporate high powers and low traverse speeds result high irradiances, corresponding to a relative increase in predicted maximum temperature. Likewise, Fig. 4 (b) suggests that small spot sizes and high powers result in an increase in maximum temperature. Fig. 4 (c) also suggests that that low traverse speeds and small spot sizes result in an increase in maximum temperature, although the qualitative correlation between Fig. 3 (c) and Fig. 4 (c) is less evident due the scale of the z-axis in the former.
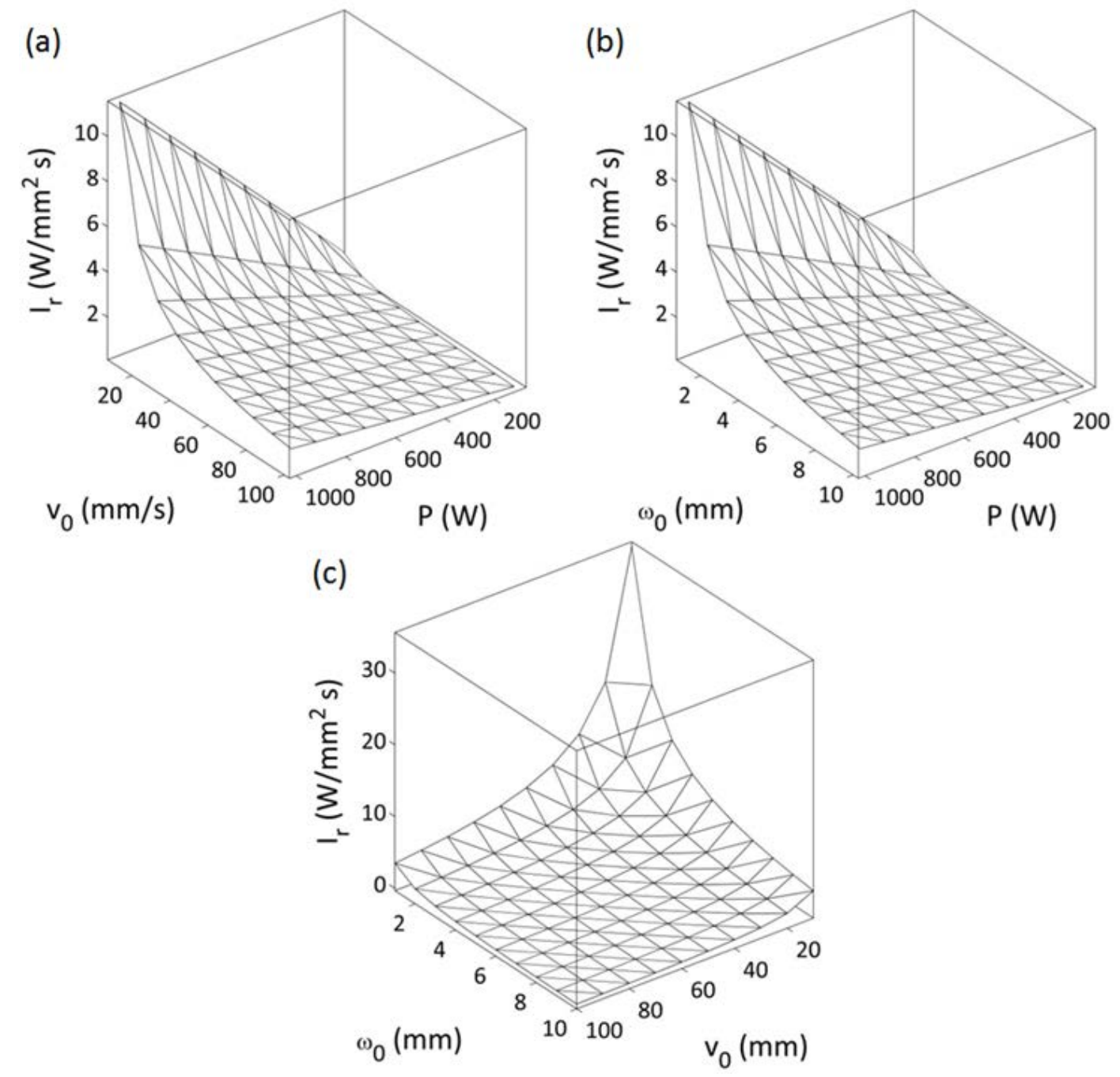

Figure 4: Laser irradiance as a function of (a) $P$ and $v_{0}\left(\omega_{0}=5.5 \mathrm{~mm}\right)$, (b) $P$ and $\omega_{0}\left(v_{0}=55\right.$ $\mathrm{mm} / \mathrm{s})$ and $(\mathrm{c}) v_{0}$ and $\omega_{0}(P=550 \mathrm{~W})$. 
Fig. 3 (d) reveals that that the Fourier number is proportional to spot size and inversely proportional to traverse speed. Low Fourier numbers are indicative of process parameters which will likely induce steep thermal gradients throughout the sheet thickness. Small spot sizes will result in a large intensities and therefore high peak temperatures. Simultaneously, high traverse speeds will limit the laser-material interaction time to such an extent that heat conduction from the irradiated top surface of the component to the bottom surface is minimized. As such, parametric combinations which incorporate high traverse speeds and small spot sizes will result in small values of $F_{0}$. Regarding the various mechanisms of laser bending discussed in the introduction, it is therefore clear that low Fourier numbers are desirable if out of plane bending towards the beam is required. It should be noted that the response curves for $F_{0}$ as a function of laser power have been omitted from Fig. 3 as the Fourier number is independent of this parameter.

Goal driven optimization for process parameter prediction. Optimal processes parameters were predicted through application of the multi-objective genetic algorithm (MOGA) in ANSYS Workbench. In this case, the simultaneous goals of the optimization process were to achieve a target $T_{0}$ value of $1400 \mathrm{~K}$ and to minimize $F_{0}$. The results of the optimization and subsequent verification (that is, candidate and verification) are shown in Table 2. Also presented in Table 2 is the postverification maximum occurring temperature on the bottom surface of the component $\left(T_{1.5}\right)$ and the maximum occurring temperature gradient between the top and bottom surfaces of the component $(\Delta T)$.

Table 2: Results of the MOGA optimization.

\begin{tabular}{|c|c|c|c|c|c|c|c|}
\hline & $P(W)$ & $v_{0}(\mathrm{~mm} / \mathrm{s})$ & $\omega_{0}(\mathrm{~mm})$ & $T_{0}(\mathrm{~K})$ & $T_{1.5}(\mathrm{~K})$ & $F_{0}$ & $\Delta T$ \\
\hline $\begin{array}{l}\text { Candidate A } \\
\text { Verification A }\end{array}$ & 189.34 & 99.74 & 1.38 & $\begin{array}{c}1140.51 \\
909.89\end{array}$ & $\begin{array}{c}- \\
598.96\end{array}$ & $\begin{array}{l}0.09 \\
0.10\end{array}$ & $\begin{array}{c}- \\
310.93\end{array}$ \\
\hline $\begin{array}{l}\text { Candidate B } \\
\text { Verification B }\end{array}$ & 263.06 & 82.17 & 1.61 & $\begin{array}{l}1139.90 \\
1078.40\end{array}$ & $\begin{array}{c}- \\
754.30\end{array}$ & $\begin{array}{l}0.12 \\
0.14\end{array}$ & $\begin{array}{c}- \\
324.1\end{array}$ \\
\hline $\begin{array}{l}\text { Candidate C } \\
\text { Verification C }\end{array}$ & 177.27 & 61.69 & 1.18 & $\begin{array}{c}1139.47 \\
943.82\end{array}$ & $\begin{array}{c}- \\
594.26\end{array}$ & $\begin{array}{l}0.13 \\
0.13\end{array}$ & $\begin{array}{c}- \\
349.56\end{array}$ \\
\hline
\end{tabular}

Of the three candidates listed in Table 2, Candidate $\mathrm{B}$ results in the highest simulated peak temperature after verification whilst maintaining a low Fourier number and therefore a steep thermal gradient, comparable to those of Candidates A and C. The higher peak temperature will result in relatively more deformation, whilst the low Fourier number will ensure that the deformation induced is out of plane and directed towards the beam, according to the TGM. Candidate B also has the advantage of a traverse speed which represents a value towards but not at the limit of the capability of the laser system intended for use. An inherently iterative process, laser bending must have a high throughput in order to compete with contact based forming processes. However, laser traverse speed is the most straightforward of the three process parameters listed in Table 2 to manipulate in-process; as such it is important that there remains the latitude for manipulating $v_{0}$. For instance, it can be beneficial during the laser bending process to speed-up upon approaching a corner or edge to avoid over heating of the component. Therefore, Candidate B is chosen as the most promising process parameter combination for subsequent experimental validation.

\section{Conclusions}

A numerical study into the intelligent prediction of process parameters for the out of plane laser bending of $80 \times 80 \times 1.5 \mathrm{~mm}$ DP 1000 steel has been conducted. Process parameters required for achieving optimal efficiency in laser bending with respect to bending mode and magnitude whilst minimizing micro-structural transformation in the heat affected zone were predicted. 
A 31 simulation numerical design of experiments was conducted, with design points chosen according to the rotatable central composite design method of design of experiments. Response surfaces were generated which revealed a clear correlation between maximum simulated temperature and laser irradiance.

Three process parameter combinations were determined through application of a multi-objective genetic algorithm. The goal of the optimization was to minimize the Fourier number whilst seeking a target maximum temperature of $1140 \mathrm{~K}$, above which the component would be susceptible to significant microstructural transformation. An optimal process parameter combination of $263 \mathrm{~W}$ laser power, $82 \mathrm{~mm} / \mathrm{s}$ traverse speed and $1.6 \mathrm{~mm}$ beam radius was chosen on the basis of peak simulated temperature and thermal gradient.

The optimization process could be improved (that is, the discrepancy between candidate and verification output values reduced) through implementation of a more rigorous sampling procedure incorporating more design points. Additionally, incorporation of transient thermal-mechanical finite element simulations would allow this technique to be utilized in closed loop, intelligent prediction systems for the iterative forming or correction of distortion in components. A key challenge remains as the optimization of numerical simulations with respect to their computational efficiency. This is essential if they are to be utilized for real-time prediction of process parameters.

\section{References}

[1] F. Vollertsen, J. Sakkiettibutra, Different types to use laser as a forming tool, Physics Procedia Part B (2010) 193-203.

[2] M. Geiger, F. Vollertsen, Model for the temperature gradient mechanism of laser bending, Proceedings of the LANE 1 (1994).

[3] R. Neugebauer, S. Scheffler, R. Poprawe, A. Weisheit, Local laser heat treatment of ultra high strength steels to improve formability, Production Engineering 3(4-5) (2009) 347-351.

[4] J. Griffiths, S. Clark, G. Sheikholeslami, S.P. Edwardson, G. Dearden, Towards optimization of beam mode for high efficiency laser thermal forming within metallurgical constraints, International Workshop on Thermal Forming and Welding Distortion (2014) 233-241.

[5] M. Safari, M. Farzin, A. Ghaei, Investigation into the effects of process parameters on bending angle in the laser bending of tailor machined blanks based on a statistical analysis, J Laser Appl 25(5) (2013).

[6] K. Maji, D.K. Pratihar, A.K. Nath, Experimental investigations and statistical analysis of pulsed laser bending of AISI 304 stainless steel sheet, Optics \& Laser Technology 49(0) (2013) 18-27.

[7] M. Omidvar, R. Fard, H. Sohrabpoor, R. Teimouri, Selection of laser bending process parameters for maximal deformation angle through neural network and teaching and learning-based optimization algorithm, Soft Computing (2014) 1-12.

[8] K. Maji, D.K. Pratihar, A.K. Nath, Laser forming of a dome shaped surface: Experimental investigations, statistical analysis and neural network modeling, Optics and Lasers in Engineering 53(0) (2014) 31-42.

[9] K. Maji, D.K. Pratihar, A.K. Nath, Analysis of pulsed laser bending of sheet metal using neural networks and neuro-fuzzy system, Proceedings of the Institution of Mechanical Engineers, Part B: Journal of Engineering Manufacture 228(9) (2014) 1015-1026.

[10] S.P. Edwardson, J. Griffiths, G. Sheikholeslami, G. Dearden, Optimization of Beam Mode for High Efficiency Laser Thermal Forming within Metallurgical Constraints, Physics Procedia 56(0) (2014) 1392-1399.

[11] W.M. Steen, Laser Benging or Forming, Laser Material Processing 3rd ed., Springer-Verlag London, 2003, pp. 302. 